\title{
Danke Mariam! oder Sprechstunde am oberägyptischen Nil
}

Andreas Knoblauch

Korrespondenz: Dr. med. Dr. h. c. Andreas Knoblauch Zentralstrasse 15 CH-9403 Goldach

anknoblauch@bluewin.ch
Das Nile Hospital in Naqada ist eine Institution der Egyptian Medical Services (EMES). Älteren Ägyptenkennern mag die Vorgängerin, das «Schweizer Spital in Assuan» noch in Erinnerung sein. Ich erinnere mich, dass meine Mutter in den 50er Jahren alljährlich um die Neujahrszeit, die wir im Katarakt Hotel verbrachten, diesem Spital einen Besuch abstattete und neue Bettwäsche brachte.

Naqada ist eine Kleinstadt 30 Kilometer nilabwärts von Luxor. Nach langen Vorarbeiten konnte 2006 das Spital eröffnet werden. Dank Spenden, Beiträgen der Schweizerischen Direktion für Entwicklungszusammenarbeit (DEZA) und dank qualitativ hochstehender Dienstleistungen konnte sich das Spital innerhalb kurzer Zeit einen ausgezeichneten Ruf erwerben.

Zur überregionalen Attraktivität hat die Beratertätigkeit von drei Schweizer Spezialärzten beigetragen, die in regelmässigen Abständen nach Naqada reisen, ihr Wissen zur Verfügung stellen und an die lokalen Spezialisten weitergeben. Der Schreibende ist einer von ihnen. Während die Krankheitsbilder in meinem Fach, Lungenmedizin, in Ägypten in etwa ähnlich sind wie in Europa, hebt sich die Kultur rund um die
Sprechstunde stark von dem ab, was wir gewohnt sind. Ein Beispiel:

Ein koptischer Priester betritt in vollem Ornat das Sprechzimmer und stützt dabei seine alte, kranke Mutter. Während die beiden Platz nehmen, vergewissere ich mich, wer sonst noch in diesem winzigen Raum ist: Da ist Dr. M., der diensttuende Klinik-Arzt. Ferner eine Assistenzärztin, die von der benachbarten Universitätsstadt gekommen ist, um vom fremden Doktor zu lernen, im Arm hat sie einen Säugling. Dann Mariam, die kleine, muntere und blitzgescheite Arztgehilfin. Nach einer Weile kommt der Laborchef dazu, wie auch der Röntgentechniker, weil sie vermuten, dass es für sie Aufträge geben wird. Jetzt wendet man sich der Patientin zu, und zuerst wird nach den Beschwerden gefragt. Ich richte meine Fragen an die kranke, leidend dreinblickende Patientin, die Antworten gibt aber der Sohn und Priester. Ich bitte Dr. M. um Übersetzung. Das führt zu einem Palaver, in das sich bald die beiden Laboranten einmischen. Schliesslich redet auch die Assistenzärztin mit, «damit die Übersetzung stimmt», wie sie mir später sagt. Übersetzt wurde aber noch gar nichts, und die alte Dame hat auch noch gar kein Wort gesagt.

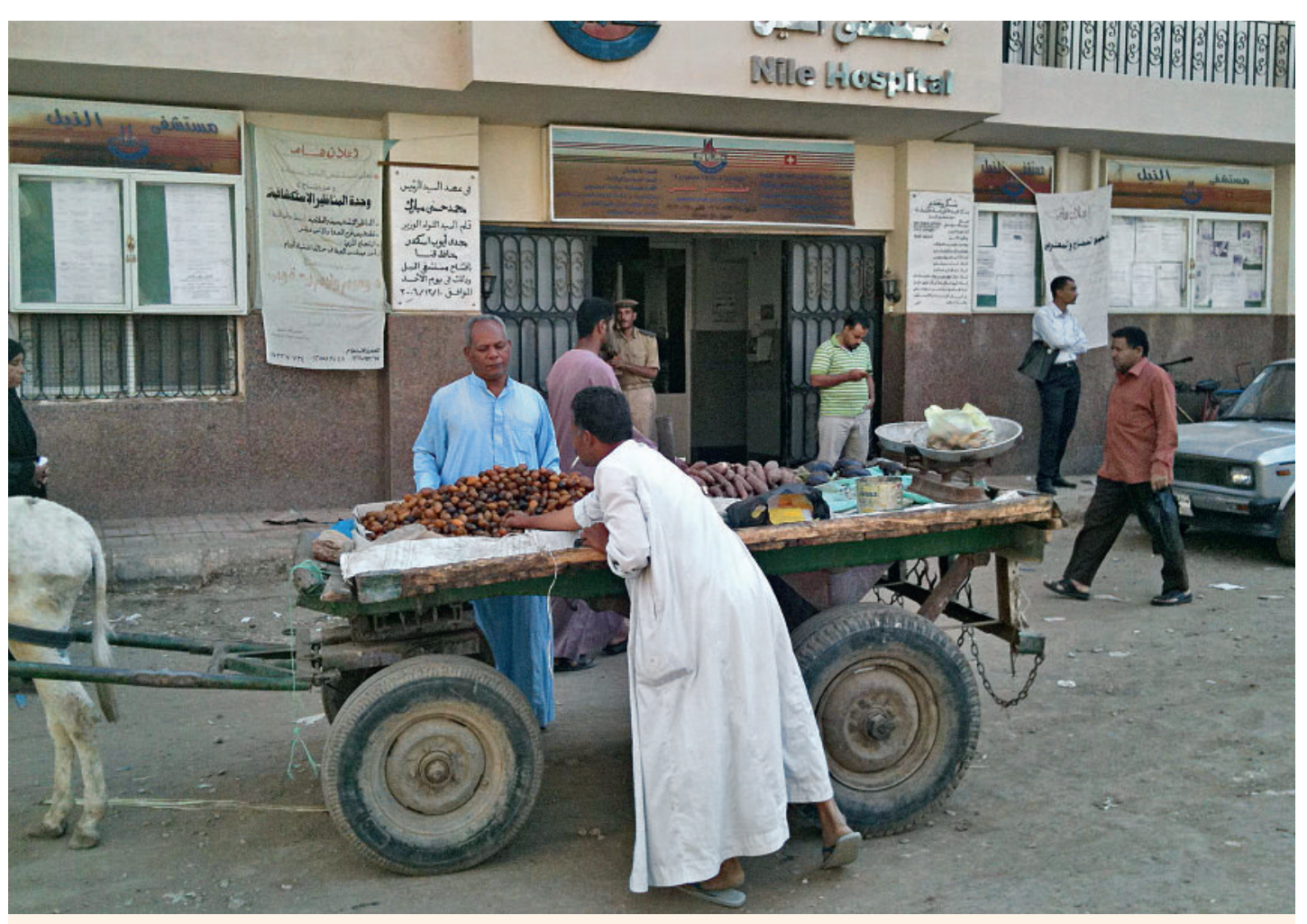

Der Eingang zum Nile Hospital in Naquada, Ägypten, in dem regelmässig auch drei Schweizer Ärzte arbeiten. 
Jetzt muss sie sich zwecks Untersuchung den Oberkörper freimachen. Das tut sie nur ungern. Mariam, die Arztgehilfin, zieht am Unterrock, ich will hinten den Büstenhalter öffnen, aber der ist vernäht. Inzwischen ist ein weiterer Sohn hinzugekommen. Die beiden Söhne halten jetzt Badetücher um die Mutter und bedecken das mühsam freigelegte Stück Haut, wobei sie geniert wegschauen. Die Assistenzärztin hat inzwischen einen Telefonanruf bekommen und bespricht sich mit ihrem Mann. Ich zähle spasseshalber die Anwesenden: neun sind es inzwischen, davon zwei lärmige Schlingel. Jetzt steht die Assistenzärztin auf und sagt, sie müsse gehen, um das Baby zu stillen. Inzwischen ist es uns gelungen, die alte Dame auf den Untersuchungstisch zu hieven, aber unglücklicherweise sitzt sie auf der Handtasche der Assistenzärztin. Die Handtasche wird unter der alten Dame hervorgewürgt. Die Assistenzärztin kämpft sich durch die vielen Menschen zum Ausgang und ist weg, ebenso ihre Fortbildung beim ausländischen Doktor.

Wir finden heraus, dass die alte Dame wirklich etwas hat, und ich mache einen Ultraschall, ein Röntgenbild. «Man darf ihr aber nicht sagen, was sie hat, das macht man in Ägypten nie», sagt mir Dr. M. hinter vorgehaltener Hand. Die Tür geht jetzt auf, und Mariam bringt türkischen Kaffee. Der Priester bespricht sich mittlerweile ebenfalls am mobilen Te-

\section{Jetzt tritt auch noch ein Mann mittleren Alters ein.}

\section{Da sich niemand wundert, tue ich es auch nicht.}

lefon, und ich unterhalte mich mit der Patientin auf Arabisch, was zu meinem Stolz ordentlich geht. Jetzt streckt mir der Priester sein Handy entgegen; ein weiterer Bruder sei dran, der wolle Auskunft. Dieser spricht gebrochen Englisch und sagt, er sei Arzt. Ich gehe aus dem Zimmer wegen zu viel «Dauscha», was Lärm heisst. Bruder Schenuda lässt sich alles erklären und dankt gerührt für die Fürsorge, die der ausländische Doktor seiner alten Mama zukommen lässt. Zurück ins Sprechzimmer: Dort findet gerade eine hitzige Auseinandersetzung wegen des weiteren Vorgehens statt. Als alles besprochen und weitschweifig erklärt ist, verabschiede ich Mutter und Sohn, die aber bleiben sitzen. Jetzt geht die Tür auf, und ein Mann mittleren Alters tritt ein. Da sich niemand wundert, tue ich es auch nicht. Jetzt steht der Priester auf, nimmt die runde Mütze vom Kopf und legt sie sorgfältig auf mein Pult. Auch das grosse Kreuz mit der goldenen Kette wird abgenommen und in die Mütze gelegt. Ich merke jetzt, dass der Priester selbst untersucht werden möchte. Niemand von den Anwesenden macht Anstalten, das Zimmer zu verlassen. Es stellt sich übrigens heraus, dass der neu Hinzugekommene ein weiterer Bruder ist. Der Priester wundert sich, dass er seinen langen Rock ausziehen soll. Der
Techniker vom Labor und ein Bruder halten ein Leintuch um seinen Körper, um jeden sichtbar werdenden Hautteil zu verstecken, was das Abhören erschwert. Unter verschämtem Gekicher schaffen wir eine Lockerung der Tücher. Mariam hat sich hinter einen Vorhang verzogen, weil es nicht schicklich ist, bei einem Priester eine nackte Stelle zu sehen. Der Priester hat tatsächlich einen Befund. Ich fülle Zettel für die weitere Untersuchung aus.

Zwei Ärzte, die gerade Flaute in ihrer Sprechstunde haben, gesellen sich zu uns. Zwischen den drei Brüdern und den drei Ärzten ist bald eine fröhliche Diskussion im Gange. Es wird herzlich gelacht. Dann gebietet Dr. M. der Runde Ruhe und sagt, zu mir gewandt: «Kennst Du den besten Witz der Woche? Du hast gestern am Telefon Deiner Frau in der Schweiz gesagt, Du seist gerne in Naqada!» Jetzt wird gelacht, Hände werden ineinander geklatscht, man spürt sich kaum mehr, so sehr schüttelt einen diese Komik: gerne in Naqada zu sein, in diesem Kaff, am Ende der Welt, wo doch hier jeder am liebsten in «Amrika» oder in «Orobba» wäre! Nachdem ausgelacht, weiterführende Witze ausgetauscht, alles endgültig besprochen und wiederholt ist, ist es schon spät, doch trotz meines Abschiedsgrusses erhebt sich niemand. Jetzt stellt sich Sohn Nummer drei vors Pult und will untersucht werden. Zum Glück liegt nichts Ernsthaftes vor. Er hat einen harmlosen Husten, wie er nach einer Grippe auftreten kann und mit oder ohne ärztliche Hilfe spontan abklingen wird. Ich erkläre wortreich, dass Medikamente in dieser Situation verschwendetes Geld seien - «ja, vom Balkon heruntergeworfenes Geld», doppelt Dr. M nach - am besten wäre es für ihn, er würde gegen den Husten gar nichts nehmen: Erstens nützten die Medikamente nichts, und zweitens riskiere er, von den Nebenwirkungen krank zu werden. Ich würde ihn deshalb ohne Rezept entlassen, im modernen Orobba denke man so, kein europäischer Arzt würde im Entferntesten daran denken, ihm einen Hustensirup zu verordnen, das nütze nur der Industrie ... damit würde ich ihm eine gute Besserung wünschen, ebenso wie der ganzen Sippe, er sei entlassen, Etfaddal, dort ist die Türe ...

Jetzt schaltet sich Mariam, die Arztgehilfin ein. Ihre Augen sind aufgerissen, sie weiss, wie sehr der Ruf des Spitals durch eine Arztvisite ohne die Verordnung eines Medikaments beschädigt würde, sie ahnt den Clash der Zivilisationen, den ich mit dieser vermeintlich billigen Lösung entfessele, das kulturelle Desaster, das sich anbahnt, sie hört in ihrer Vorstellung bereits die Sippe murren, dieser Arzt sei nicht einmal fähig, ein Rezept auszustellen. Sie stellt sich kerzengerade auf, ja, jetzt hat sie den Rezeptblock gleich selbst in die Hand genommen, sie hat den Kugelschreiber schon in der Hand: «Ya doctor, soll ich einen Hustensirup aufschreiben?», fragt sie freundlich. Müde vom Tag lehne ich mich zurück, «okay» hauche ich, gerettet ...

Danke, Mariam! 